\title{
SM Otolaryngology Numerical Survey of the Different Shapes of Human's Ear Concha
}

\author{
Article Information \\ Received date: Nov 07, 2016 \\ Accepted date: Jan 10, 2017 \\ Published date: Jan 18, 2017 \\ *Corresponding author
}

Abraham Tamir, Emeritus Professor of Chemical Engineering, Ben-Gurion University of the Negev, Israel, Email: atamir4@012.net.il

Distributed under Creative Commons CC-BY 4.0

Article DOI 10.36876/smotol.1002

\section{OPEN ACCESS}

ISSN: 2574-2418

\author{
Abraham Tamir ${ }^{1 *}$ \\ ${ }^{1}$ Emeritus Professor of Chemical Engineering, Ben-Gurion University of the Negev, Israel
}

\section{Abstract}

The major aim of this article is to report experimental results about the numerical quantities of the different shapes of human are ear concha as well as to classify them into defined shape groups. To the best of the author knowledge this approach has never been applied. The results are based on 2425 pictures of ears. The major results are: (1) The author has succeeded to classify all ears concha into 36 groups demonstrated in Figure 2 . On the left is the absolute number of concha of a certain type where on the right is the percentage of it. (2) The most widespread concha, number 29 , is that which appeared 393 times and is $16.2 \%$ of the total number. The less widespread shape, number 34 , appears one time namely $0.04 \%$ of the total. In addition to the numerical results the following subjects are elaborated with respect to human's ear: its anatomy, its different shapes as well as their relation to human's character.

\section{Introduction}

\section{Ear Function and Anatomy}

Hearing, the most important function of the ear is a process of detecting air vibrations, the sound, where anatomically the ear, in combination with the brain, is the organ of hearing. It is demonstrated humorously in Figure 1-right. The human ear can nominally hear sounds in the range $20 \mathrm{~Hz}$ to $20,000 \mathrm{~Hz}(20 \mathrm{kHz})$. The upper limit tends to decrease with age where most adults being unable to hear above $16 \mathrm{kHz}$. The word "ear" may be used correctly to describe the entire organ or just the visible portion shown in Figure 1. The ear's role is to convert sound waves into nerve impulses, which are transmitted to the brain where they are interpreted as sounds. The ears, besides being a high fidelity stereophonic hearing system, are also responsible for keeping the body in balance. This is done with the help of two systems located within the inner ear. The ear consists of external, middle, and inner structures [1,2]. The external ear gathers sound waves and directs them to the eardrum. The eardrum vibrates when sound waves strike it. In the middle ear three small bones that move with that vibration and translate the vibration energy to mechanical energy, which it transferred to the cochlea-the inner ear. In the cochlea the energy becomes pressure waves in a fluid. These pressure wave move through the cochlea (shaped like a snail shell) and trigger nerve endings called hair cells, which are sensitive to different wavelengths. Each stimulated hair cell sends a nerve signal to the brain, which processes the whole as sound. Figure 1 demonstrates the external ear anatomy. The concha, analyzed numerically in the article, is the hollow bowl-like portion of the external ear that leads to the canal. The concha [3], with a volume approximately of 4 $\mathrm{cm}^{3}$ is a broad shallow cavity that is tightly coupled to the canal. This cavity is partially divided by the

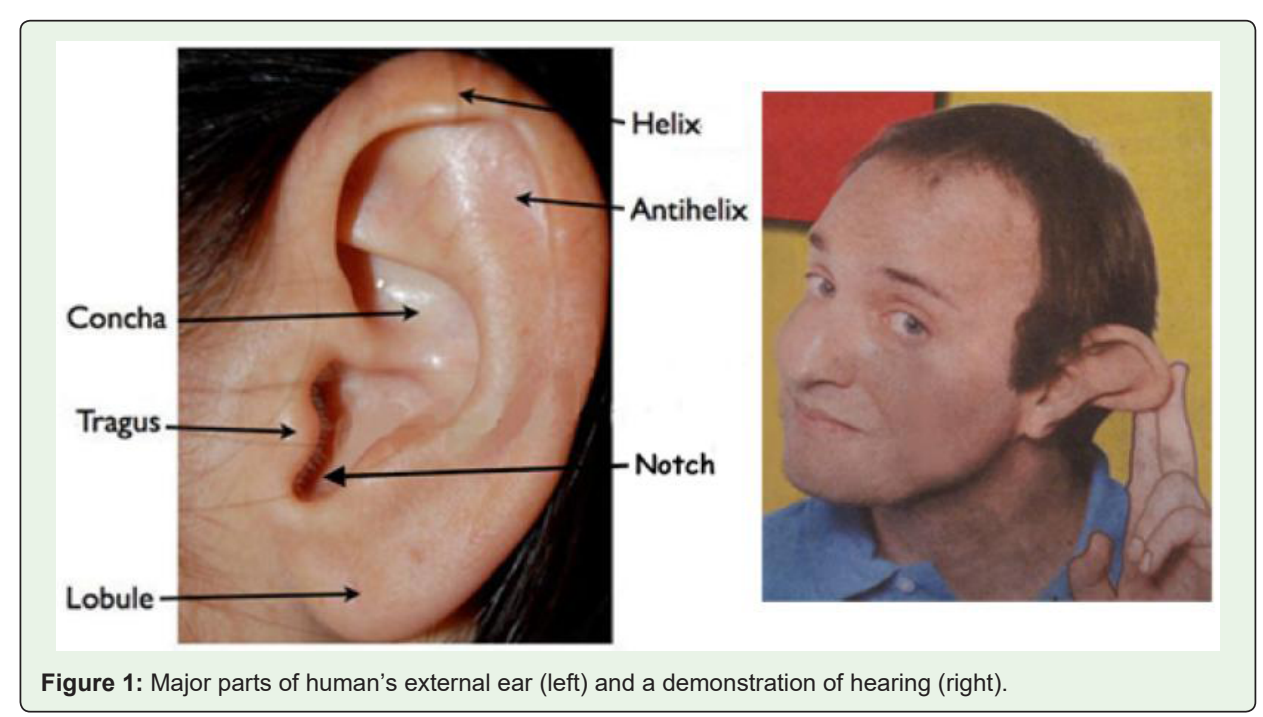


crus helias and the upper part, the cymba, is connected to the fossa. These features clearly have specific acoustical attributes whereas the structures extending from the concha, such as the helix, the anti helix, and the lobule, seems to function collectively as a simple flange. The helix is the outer frame of the ear that is a rolled up edge. The anti helix is a "Y" shaped part of the ear. The tragus is a small projection just in the front of the ear canal. The Lobule is the fleshy lower portion of the ear where the External Auditory Meatus is a tube running from the outer ear to the middle ear through which sound enters the ear. An important remark about human ears is concerning the fact that we have two ears. The reason is because they form a stereo system allowing us to identify the place from which the sound originated and also to identify whether the source is moving. The processing of sound by the ear and brain is so accurate that clicks presented to one ear and then the other can be distinguished as separate sounds when the time interval between them is as small as thirty microseconds. To conclude the introduction we may say that the human ear is a great masterpiece of engineering constructed by the Creator of mankind.

\section{Shapes of human's ear}

Before analyzing numerically the ear concha shapes it is interesting to note that the shape of the ear may indicate about the human character [4]. Ears close to head characterize a person that plans ahead, is thrifty and does not like to leave too much to chance. Protruding ears characterize a dominant personality of a leader, an original thinker, lonely and nonconformist. Ears hanging loosely characterize a tired person, a little bit embittered and not ambitious. Ears with small lobules characterize dependant personality that may lack initiative. Ears with large lobules belong to independent and strong-minded person where large ears characterize an intellectual person. Ears with no lobules characterize unresponsive person who

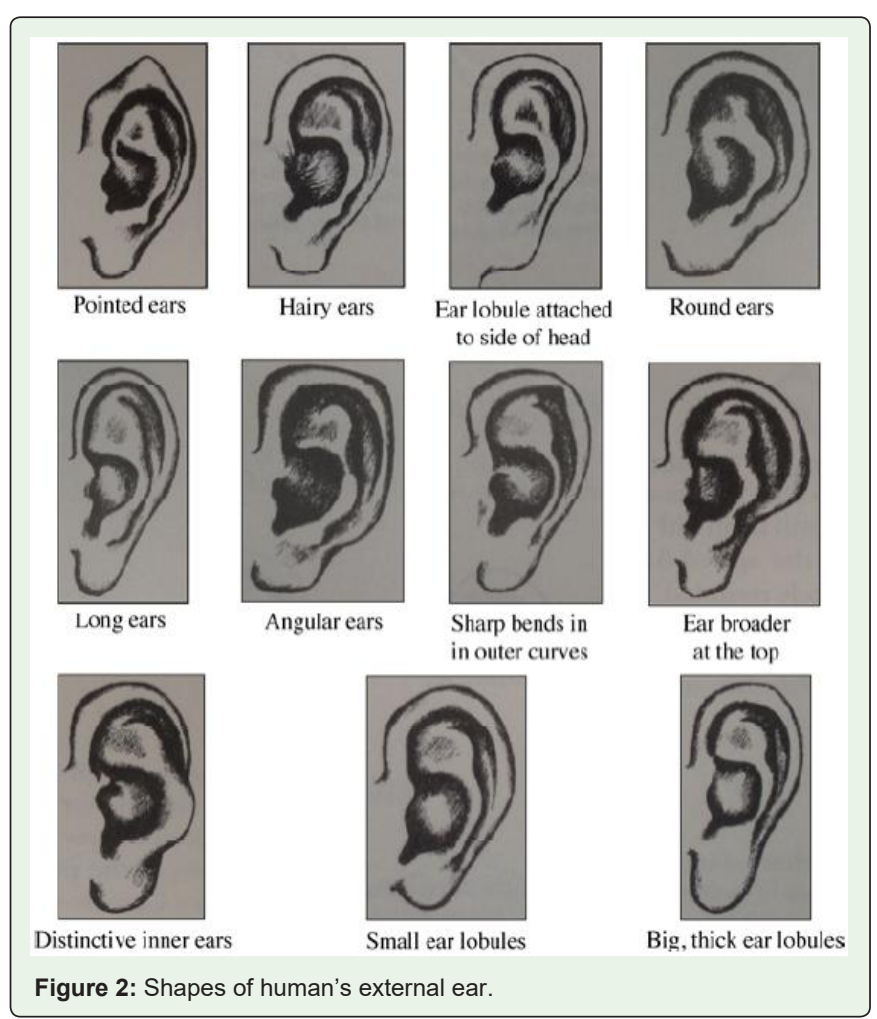

lacks purpose. Longer than wider ears indicate a keen and impractical personality where small ears indicate instinctive. And finally elliptical ears belong to stubborn people and initiators [5].

A more detailed relationship between ear shape and human character based on 11 ears is based on Figure 2 reported in [6]. "Pointed ears" belong to people who lack feelings and sympathy. However they are known to be original and highly inventive and imaginative. "Hairy ears" are self-opinionated, eager to learn, often extravagant, rarely uses own talents. "Ear lobules attached to the side of head" characterize egotistical, little depth of feeling, behaves superficially and likes to go in search of adventure. "Round ears" are friendly disposition, trustworthy, optimistic, sociable and dreamy. "Round ears" are friendly disposition, trustworthy, optimistic, sociable and dreamy. "Long ears" have great intellect, lays claim to wisdom and high morals and prefers to be distant. "Angular ears" is shrewd, vivacious, disposition quick intelligence and needs constant diversion. "Sharp bends in outer curves" is obstinate, stubborn, workhappy, many talented, must cope with great family misfortunes. "Ear broader at the top" is naïve, adaptable, easily pleasant and hedonistic. "Distinctive inner ear" has a bundle of energy, creative, unconventional with tendency to exaggerate. "Small ear lobules" is emotional, get involved in violent arguments, quickly loses patience and works unsystematically. "Big with thick ears lobules" is domineering, goal-oriented and permanently in search of ideals. And finally it is interesting to mention that no information was found relating ear concha shape and human character. Numerical results of ear concha shapes the major source to investigate ears concha shape were pictures of ears photographed in Israel by the author, about 95\% of all data. Based on [7] the author succeeded to classify all the 2425 ears into 36 groups demonstrated in Figure 3. The left figure is the total number of ears of a certain shape for men and women together.

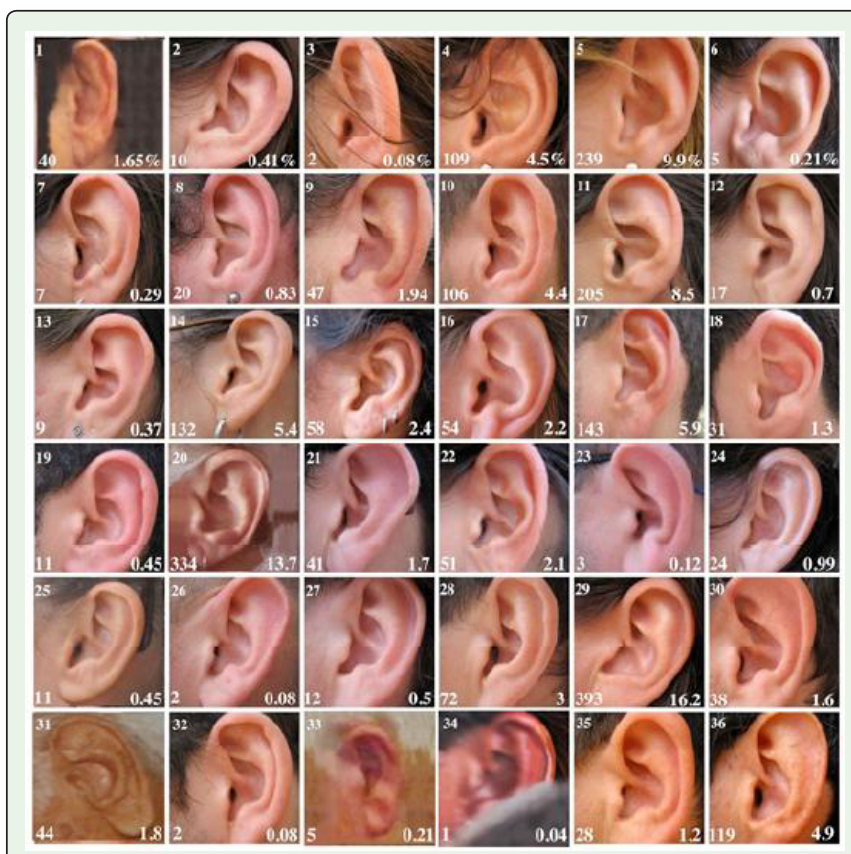

Figure 3: Numerical results of all shapes of ears concha based on 2425 ears of men and women. Left figure is the total number of ears of a certain shape for men and women together where the right figure indicates the percent of this shape from the total number of ears. 


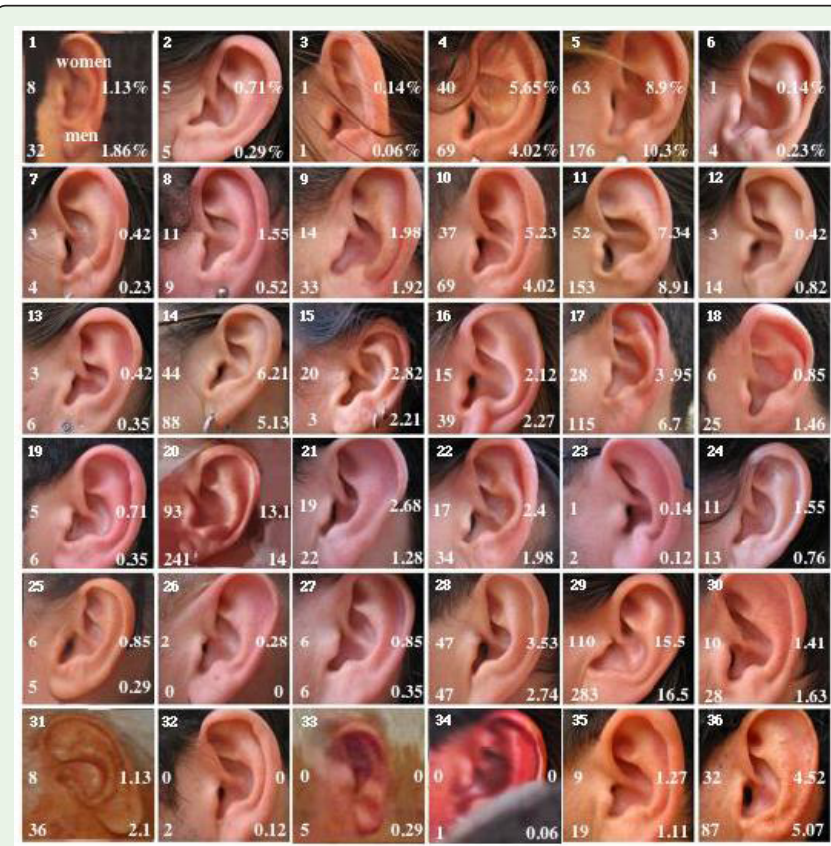

Figure 4: Numerical results for men (bottom two numbers) and women (middle two numbers) of the different shapes of ear concha based on 2425 ears. Left number is the total number of ears of the type in the figure. Right number is the percent of this type from the total number of ears for 1715 men and 708 women.

The right figure indicates the percent of this shape from the total number. Figure 4 is a more detailed report. The bottom two numbers are for men and the middle numbers are for women. Left number is the total number of ears of the type in the figure where the right number is the percent of this type from the total number of ears for 1717 men and 708 women. It should be noted that shape 34 is unique and the only one existing among the 2425 ears.

A deep look at all ears in Figure 3 reveals that in principle there are two basic shapes of the ear concha. The first one is without a notch at the lower part of the concha: types 1,20,29, 31 and 36, where in the rest there is a notch. Type 29 may be looked upon as the beginning of a notch. As indicated before no relation exists between human character and the ear concha shape.

\section{Conclusion}

The major results and conclusions of the ears collection and their classification may be summarized as follows:

1) The most widespread concha shape is number 29 that appears 393 times, $16.2 \%$ of all ears investigated. Of this shape $16.5 \%$ characterize the 1715 men and $15.5 \%$ present the 708 women.

2) Ear concha 34, of a man, appears only once!

3) The notch has a different shape and length where in principle there are relatively long, narrow and wide notches; however a precise classification is impossible.

In conclusion the author believes that the ears shapes in Figure 3 based on analysis of 2425 ears as well as the numerical analysis of the results gives a more precise information about the reality. The latter might also enhance a more concentrated analysis and research of what the ear, its concha and notch shapes and size reveals in our face. And finally it is interesting to emphasize the fact that there exist also artificial ears. This is a device designed to duplicate the frequency response, acoustic impedance, threshold sensitivity, and relative perception of loudness, consisting of a special microphone enclosed in a box with properties similar to those of the human ear.

\section{References}

1. http://library.thinkquest.org/22484/noframes/ears_.html

2. http://wiki.answers.com/Q/What_are_the_ear_functions_and_how_do_they_ work

3. http://books.google.co.il/books?id=1x_RvffWhcC\&pg=PA1093\&lpg=PA109 $3 \& d q=$ function+of+ear+concha\&source $=$ bl\&ots $=$ aNL8XxzN\&sig $=2 X Y$ fxrrS TGLBUJjejufBZzUM5A\&hl=iw\&ei=IsZwTPKuBNKIOMa1rLAL\&sa=X\&oi=bo ok_result\&ct=result\&resnum $=4 \& v e d=0 C B w Q 6 A E w A z g K \# v=$ onepage $\& q=$ fun ction $\% 20$ of $\% 20$ ear $\% 20$ concha\&f false

4. http://astrology.com.au/wisdom/face-reading

5. http://www.shawnfeeney.com/sketch.php?sketchpage $=24$

6. Chi an Kuei. "Face Reading". Published by M. Evans and Company, Inc. 1998.

7. http://www.tele-smart.com/blog/wp-content/uploads/2010/02/collage-ofears1.jpg 